\title{
Langevin Forces and Generalized Transfer Fields for Noise Modelling in Deep Submicron Devices
}

\author{
P. SHIKTOROV ${ }^{\mathrm{a}, *}$, E. STARIKOV ${ }^{\mathrm{a}, \mathrm{d}}$, V. GRUZ̃INSKIS ${ }^{\mathrm{a}}$, T. GONZÁLEZ ${ }^{\mathrm{b}}$, J. MATEOS ${ }^{\mathrm{b}}$, \\ D. PARDO ${ }^{\mathrm{b}}$, L. REGGIANI ${ }^{\mathrm{c}}$, L. VARANI $^{\mathrm{d}, \uparrow}$ and J. C. VAISSIEREE ${ }^{\mathrm{d}}$ \\ ${ }^{a}$ Semiconductor Physics Institute, Vilnius, Lithuania; ${ }^{\mathrm{b}}$ Universidad de Salamanca, Salamanca, Spain; \\ ${ }^{\mathrm{c}}$ Università di Lecce, Lecce, Italy; ${ }^{\mathrm{d}}$ Université Montpellier II, 34095 Montpellier Cedex 5, France
}

We present a generalized transfer field method with the microscopic noise sources directly connected with the velocity and energy change during single scattering events. The advantages of this method are illustrated by hydrodynamic calculations of current and voltage noise spectra in several two-terminal submicron structures.

Keywords: Noise; Modeling; Hydrodynamics; Submicron devices

\section{INTRODUCTION}

A standard technique to compute noise in electronic devices is the impedance field method $[1,2]$, a deterministic approach able to provide analytical and numerical solutions for the noise spectra of electronic devices at a hydrodynamic level [3]. However, even in its advanced form [2] this method is not appropriate for deep submicron devices where spatial correlations between noise sources cannot be neglected [4]. To overcome these difficulties, we have recently developed a new scheme [5] where the noise sources are directly connected with the velocity and energy changes during single scattering events. The aim of this work is to implement the new scheme within a generalized transfer field (GTF) method and present the corresponding computational procedures which are then applied to noise calculations in several submicron structures such as GaAs $n^{+} n n^{+}, n^{+}-n$-Schottky-contact and $\mathrm{Si} p^{+} n$ diodes operating under various conditions including voltage, current, and circuit-noise operation modes.

\section{GENERALIZED TRANSFER FIELD METHOD}

The GTF relates macroscopic fluctuations of the device characteristics under interest (e.g., current, voltage, etc.) to microscopic fluctuations of the dynamical variables of a single carrier (e.g., velocity, energy and higher order moments of

\footnotetext{
*Present address: Semiconductor Physics Institute, Goshtauto 11, 2600 Vilnius, Lithuania. Tel.: (3702) 614920, Fax: (3702) 627123, e-mail: jane@pav.pfi.lt

${ }^{\dagger}$ Corresponding author. Tel.: +33467143822, Fax: +33467547134, e-mail: varani@cem2.univ-montp2.fr
} 
carrier momentum p) which are originated by single scattering events of carriers inside the device. At the kinetic level of description, the fluctuations of the carrier distribution function in momentum space due to random scattering events are described by the microscopic Langevin force, $\xi(\mathbf{p}, x, t)$. At the hydrodynamic (HD) level of description, the fluctuations of average dynamic variables such as velocity $v(x, t)$, energy $\varepsilon(x, t)$, etc., are described by the HD Langevin force, $\xi_{\alpha}=\int \alpha(\mathbf{p}) \xi(\mathbf{p}, x, t) d \mathbf{p}$, where $\alpha=v, \varepsilon, e t c$. Within the Green-function formalism, the fluctuation of any HD $H$-characteristic of a two-terminal device caused by the HD Langevin force can be represented as (for simplicity let us consider a one-dimentional geometry):

$$
\begin{array}{r}
\delta H(x, t)=\sum_{\alpha} \int_{0}^{L} d x_{0} \int_{0}^{\infty} d \tau G_{H}^{\dot{\alpha}}\left(x, x_{0}, \tau\right) \\
\xi_{\alpha}\left(x_{0}, t-\tau\right)
\end{array}
$$

Here $H(x, t) \equiv\{n(x, t), v(x, t), \varepsilon(x, t), E(x, t), \ldots\}$ can represent various local physical quantities such as carrier concentration, average velocity, average energy, conduction current density, electric field, electrical potential, etc., $G_{H}^{\dot{\alpha}}\left(x, x_{0}, \tau\right)$ is the singleparticle Green-function which describes the linear response of the $H$-characteristic at point $x$ and time $t$ to a local perturbation of the dynamical variable $\alpha$ of a single particle at point $x_{0}$ and time $t-\tau$, and $L$ is the device length. Owing to the $\delta$ like correlation of the Langevin forces both in time and space, the spatio-temporal dependence of the auto-correlation $\left(H=H^{\prime}\right)$ and cross-correlation $\left(H \neq H^{\prime}\right)$ functions of fluctuations of any two $H$-characteristics takes the form:

$$
\begin{aligned}
& C_{H H^{\prime}}\left(x^{\prime}, x^{\prime \prime}, s\right)= \overline{\delta H\left(x^{\prime}, t\right) \delta H^{\prime}\left(x^{\prime \prime}, t-s\right)} t \\
&= \frac{1}{2} \sum_{\alpha, \beta} \int_{0}^{L} d x_{0} n_{s} S_{\dot{\alpha} \dot{\beta}}\left(x_{0}\right) \\
& \int_{0}^{\infty} G_{H}^{\dot{\alpha}}\left(x^{\prime}, x_{0}, \tau\right) \\
& G_{H^{\prime}}^{\dot{\beta}}\left(x^{\prime \prime}, x_{0}, \tau+s\right) d \tau
\end{aligned}
$$

where $S_{\dot{\alpha} \dot{\beta}}\left(x_{0}\right)$ is the single particle spectral density of the Langevin force and $n_{s}\left(x_{0}\right)$ the carrier concentration pertaining to stationary conditions. By neglecting carrier-carrier scattering $S_{\dot{\alpha} \dot{\beta}}(x)$ takes the form:

$$
\begin{aligned}
S_{\dot{\alpha} \dot{\beta}}(x)=\frac{2}{n_{s}(x)} \int d \mathbf{p} \int d \mathbf{p}^{\prime}\left[\alpha(\mathbf{p})-\alpha\left(\mathbf{p}^{\prime}\right)\right] \\
\\
{\left[\beta(\mathbf{p})-\beta\left(\mathbf{p}^{\prime}\right)\right] W\left(\mathbf{p}, \mathbf{p}^{\prime}\right) f_{s}\left(\mathbf{p}^{\prime}, x\right) }
\end{aligned}
$$

where $\alpha, \beta=v, \varepsilon$, etc., $f_{s}(\mathbf{p}, x)$ is the steady-state distribution function normalized to carrier concentration at point $x$, and $W\left(\mathbf{p}, \mathbf{p}^{\prime}\right)$ is the collision rate due to all scattering mechanisms.

In the general case of a local $H(x, t)$-characteristic such as the local conduction current, $j(x, t)=e n(x, t) \nu(x, t)$, the local electric field, $E(x, t)$, etc., the correlation function given by Eq. (2) and its Fourier transform will depend on a pair of local coordinates, $\left(x, x^{\prime}\right)$. Usually one is interested in fluctuations of global $H(t)$-characteristics, such as, the total current density $(H \equiv J=$ $\left.(e / L) \int_{0}^{L} n(x, t) v(x, t) d x\right)$ or the voltage drop between the terminals $\left(H \equiv U=\int_{0}^{L} E(x, t) d x\right)$ which are already some integrals of the corresponding local characteristics over the whole device. Accordingly, the response functions of these global characteristics and their Fourier transform will depend only on $x_{0}$. By applying the WienerKhintchine theorem to the fluctuations of a global characteristic one obtains the GTF formula in the form:

$$
\begin{gathered}
S_{H}(\omega) \equiv 2 \int_{-\infty}^{\infty} \overline{\delta H(t) \delta H(t+s)} t \exp (i \omega s) d s \\
=\sum_{\alpha, \beta} \int_{0}^{L} d x_{0} n_{s}\left(x_{0}\right) S_{\dot{\alpha} \dot{\beta}}\left(x_{0}\right) \\
F_{H}^{\dot{\alpha}}\left(x_{0}, \omega\right) F_{H}^{\dot{\beta} *}\left(x_{0}, \omega\right)
\end{gathered}
$$

where

$$
F_{H}^{\dot{\alpha}}\left(x_{0}, \omega\right)=\int_{0}^{\infty} d s \exp (-i \omega s) G_{H}^{\dot{\alpha}}\left(x_{0}, s\right)
$$


is the generalized transfer field determined as the Fourier transform of the function $G_{H}^{\dot{\alpha}}\left(x_{0}, s\right)$ which gives the linear response of the global $H(t)$ characteristic of the whole device (i.e., the conduction current flowing through the structure or the voltage drop between the structure terminals) to perturbations of velocity, energy and higher order moments $(\alpha=\nu, \varepsilon$, etc.) appeared at point $x_{0}$. In the case when fluctuations of local characteristics are considered, the spectral density of fluctuations takes a form similar to Eqs. (4) and (5), however $S_{H H^{\prime}}\left(x, x^{\prime}, \omega\right)$ will describe the crosscorrelation of fluctuations in two local points $x$ and $x^{\prime}$, and $F_{H}^{\dot{\alpha}}\left(x, x_{0}, t\right)$ will describe the response of the local $H(x, t)$-characteristic at point $x$ to a perturbation of velocity, energy, etc., at point $x_{0}$.

\section{COMPUTATIONAL PROCEDURES}

The above approach is sufficiently general and can be applied to various HD models. Below we shall apply it in the framework of the velocity and energy conservation equations. Accordingly, microscopic fluctuations of carrier velocity and energy will form the basis of noise sources.

\section{Model}

The HD model includes the continuity equation for carrier concentration $n(x, t)$ as well as the conservation equations for carrier average velocity $v(x, t)$ and mean energy $\varepsilon(x, t)$ written in the form [5]:

$$
\begin{gathered}
\frac{\partial n}{\partial t}+\frac{\partial n v}{\partial x}=0 \\
\frac{\partial v}{\partial t}+v \frac{\partial v}{\partial x}+\frac{1}{n} \frac{\partial}{\partial x}\left(n Q_{v}\right)-e E m^{-1}+v \nu_{v}=\frac{1}{n} \xi_{v} \\
\frac{\partial \varepsilon}{\partial t}+v \frac{\partial \varepsilon}{\partial x}+\frac{1}{n} \frac{\partial}{\partial x}\left(n Q_{\varepsilon}\right)-e E v+\left(\varepsilon-\varepsilon_{t h}\right) \nu_{\varepsilon}=\frac{1}{n} \xi_{\varepsilon}
\end{gathered}
$$

Depending on the task to be solved, the Poisson equation for the self-consistent electric field $E(x, t)$ and the equations describing an external circuit can be added to Eqs. (6)-(8).

\section{Parameters}

Equations (7) and (8) contain five energy parametric dependencies, namely, the average of the reciprocal effective mass in the field direction $m^{-1}$, the velocity and energy relaxation rates, $\nu_{v}$ and $\nu_{\varepsilon}$, the variance of velocity-velocity fluctuations, $\mathrm{Q}_{v}=\left\langle\delta v^{2}\right\rangle_{0}$ and the covariance of velocity-energy fluctuations, $Q_{\varepsilon}=\langle\delta v \delta \varepsilon\rangle_{0}$, where brackets mean average over the distribution function and the subscript 0 indicates steady-state conditions pertaining to constant and homogenous applied electric fields. All the parameters are assumed to depend only on the local mean energy, and as such they can be obtained from a stationary Monte Carlo (MC) simulation of the bulk semiconductor [3].

\section{Noise Source}

The white spectral density of the Langevin force $S_{\dot{\alpha} \dot{\beta}}$ is also treated as depending on the local mean energy only. This quantity is determined, within a MC simulation, in parallel with the calculations of the other parameters of the HD model by averaging over a trajectory of the single-carrier random walk in momentum space as [5]:

$$
S_{\dot{\alpha} \dot{\beta}}=\frac{2}{T} \sum_{i=1}^{N} \Delta \alpha_{i} \Delta \beta_{i}
$$

where $\Delta \alpha_{i}, \Delta \beta_{i}$ are the instantaneous variations of velocity and energy of a single carrier during the $i$ th scattering event and $T$ is the total time elapsed during $N$ scattering events.

\section{Green Functions}

Calculations of the Green-functions are performed in two main steps. Firstly, in the absence of 
perturbations due to the Langevin forces, one should obtain a stationary solution of Eqs. (6) - (8) coupled with the Poisson equation and, if necessary, another equations describing the external circuit. Then, a perturbation of the steady-state values of velocity or energy, given by $\Delta \alpha \delta\left(x-x_{0}\right)$, is introduced at time $t=0$ and point $x_{0}$. Usually, the spatial profile of the perturbation is given by some approximation of the $\delta$-function which takes some volume in $x$-space, as for example a Gaussian function. The perturbation amplitude $\Delta_{\alpha}$ is taken sufficiently small to fulfill the requirement of linearity of the response. Then, a direct numerical solution of the system of Eqs. (6)-(8) jointly, if necessary, with the Poisson and circuit equations is carried out to give the relaxation of the system to the stationary state. The Greenfunction corresponding to the given $H$-characteristic is obtained from the difference between the local values of $H\left(x, x_{0}, t\right)$ calculated during the relaxation process and the values of $H_{s}(x)$ corresponding to steady-state conditions. The values of the difference are then normalized to the amplitude of the initial perturbation $\Delta_{\alpha}$ and to $n_{s}\left(x_{0}\right)$ as:

$$
G_{H}^{\dot{\alpha}}\left(x, x_{0}, t\right)=\frac{1}{\Delta_{\alpha} n_{s}\left(x_{0}\right)}\left[H\left(x, x_{0}, t\right)-H_{s}(x)\right]
$$

where $\alpha=v, \varepsilon$, respectively.

\section{NUMERICAL RESULTS}

Below the general procedure of noise calculations is applied to several submicron semiconductor structures.

\section{$n^{+} n n^{+}$Structures}

Figure 1 shows the contributions of microscopic noise sources connected with velocity and energy rates to the total value of the spectral density of voltage fluctuations calculated for a $0.21-0.30$ $0.39 \mu \mathrm{m} n^{+} n n^{+}$GaAs structure. Results obtained within the GTF method are shown together with

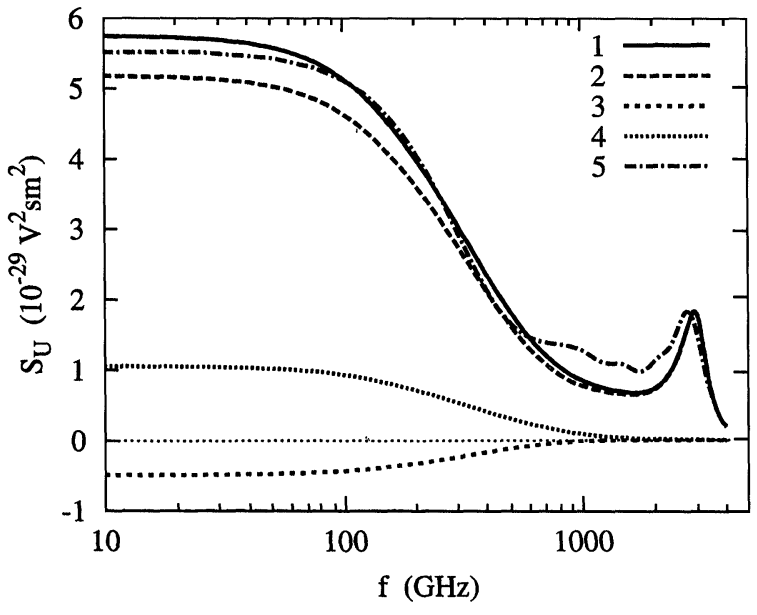

FIGURE 1 Spectral density of voltage fluctuations calculated with the GTF method for a $0.21-0.30-0.39 \mu \mathrm{m} n^{+} n n^{+}$GaAs structure with doping levels $n=5 \times 10^{15} \mathrm{~cm}^{-3}$ and $n^{+}=$ $10^{17} \mathrm{~cm}^{-3}$ at $T=300 \mathrm{~K}$ for a voltage of $0.5 \mathrm{~V}$ (curve 1). Curves 2 to 4 present, respectively, contributions coming from velocity-velocity, velocity-energy and energy-energy microscopic noise sources. Curve 5 shows the result of direct simulations of voltage noise with the MC method.

those of a direct simulation performed with the MC method. We find that the main contribution comes from the microscopic noise source related to velocity rates (curve 2). The cross-correlation between velocity and energy rates is found to give a negative contribution which partially compensates the positive contribution belonging to the autocorrelation of energy rate.

\section{Schottky Diode}

The results of noise calculations for a 0.35 $0.35 \mu \mathrm{m}$ GaAs $n^{+}-n$-Schottky-contact structure are presented in Figures 2 and 3. Since here current noise is of most interest, calculations are performed directly for current fluctuations, i.e., taking $H=J$. In so doing, intermediate quantities such as transfer field, local contributions to noise, etc., useful for a spatial analysis of the noise, are obtained in a natural way. Furthermore, by using the microscopic noise sources originated by scattering events one needs to consider just the noise sources inside the structure, thus avoiding the introduction of bulk and surface noise sources 


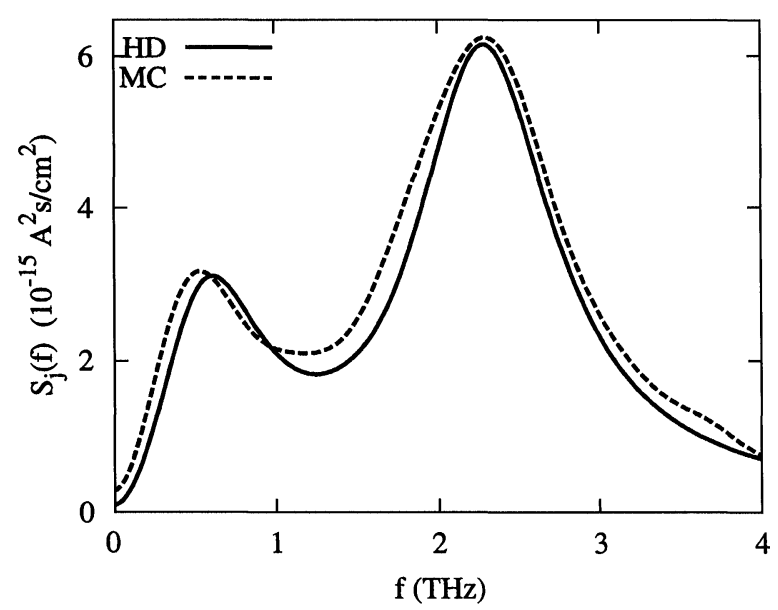

FIGURE 2 Spectral density of current fluctuations for a $0.35-0.35 \mu \mathrm{m} \mathrm{GaAs} n^{+}-n$-Schottky-barrier structure with $n^{+}=10^{17} \mathrm{~cm}^{-3}, n=10^{16} \mathrm{~cm}^{-3}$ and $U_{d}=0.575 \mathrm{~V}$. Continuous (dashed) curves refer to GTF method (MC) calculations, respectively.

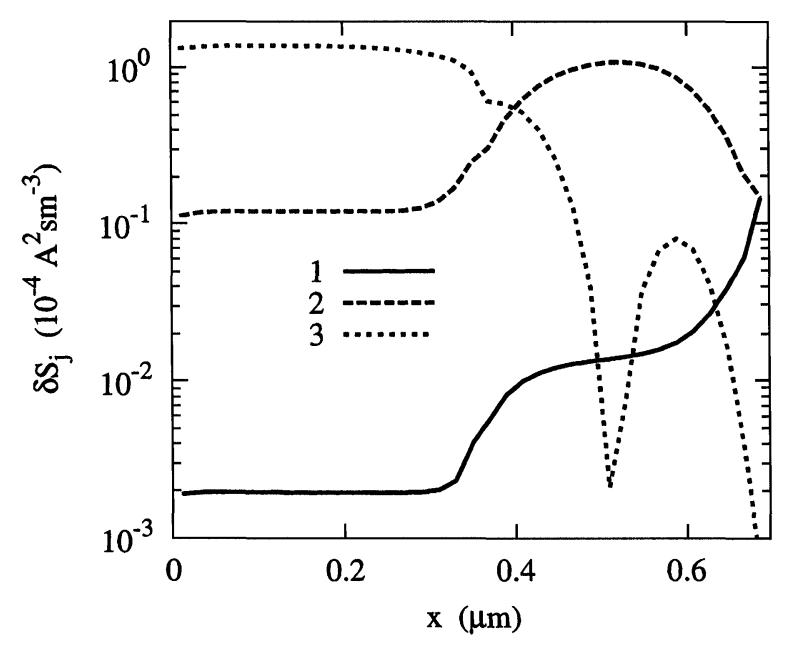

FIGURE 3 Spatial profiles of local contribution to the total current noise. Calculations refer to the GTF method under constant voltage operation mode for the Schottky-barrier structure of Figure 2. Curves 1 to 3 correspond, respectively, to frequencies $f=0,500,2500 \mathrm{GHz}$.

to describe the total noise of the device. Figure 2 compares the spectral densities of current fluctuations calculated by the GTF method and the MC procedure at $U=0.575 \mathrm{~V}$ (solid and dashed lines, respectively). As noise source, only the $S_{\dot{i} \dot{v}}$-term is accounted for. This term is found to be sufficient to describe the noise spectrum with a good accuracy practically in the whole frequency range. Accordingly, the shot-noise at low frequencies, the spike in the intermediate frequency range near $f=600 \mathrm{GHz}$ corresponding to returning carriers, as well as the plasma peak in the high-frequency range near $f=2.2 \mathrm{THz}$ are well reproduced. To emphasize the advantage given by the GTF method in providing the spatial analysis of the noise contribution, Figure 3 presents the spatial profiles of the local contribution to the conduction current noise, $\delta S_{J}\left(x_{0}, \omega\right)=n\left(x_{0}\right)\left|F_{J}^{\dot{v}}\left(x_{0}, \omega\right)\right|^{2} S_{\dot{v} \dot{v}}\left(x_{0}\right)$, calculated, respectively, at frequencies $f=0,500$, $2500 \mathrm{GHz}$ (curves 1 to 3). At low-frequency, the local contribution reaches a maximum value just near to the Schottky barrier, thus confirming that this space region is responsible for shot noise. At intermediate frequency, $\delta S_{J}$ exhibits a clear peak near to the center of the $n$-region which is associated with the returning carrier effect. At high frequency, $\delta S_{J}$ exhibits a plateau in the $n^{+}$ region and some peaks in the $n$-region which are reminiscent of the formation of standing waves due to perturbation reflection from the barrier.

\section{Bipolar Diode}

In this case the balance Eqs. (6) to (8) written separately for holes and electrons are used jointly with the Poisson equation. By introducing separate noise sources in the velocity and energy conservation equations, the GTF method is found to provide natural calculations of noise. Due to the short length of the structure, generation-recombination processes are neglected and, as noise sources, only the $S_{\dot{v} \dot{v}}$-term is accounted for. Figure 4 presents the spectral density of hole (curves 1 to 3 ) and electron (curves 4 to 6) current fluctuations calculated by the GTF method for a bipolar $0.3-0.4 \mu \mathrm{m} \mathrm{Si} p^{+} n$ structure. Analogously to the case of the Schottky-barrier diode considered above, the low-frequency $(f<1 \mathrm{GHz})$ plateau corresponding to shot noise is well reproduced for both hole and electron current noise. The numerical values well agree with the usual relation $S_{J}(0)=2 e J$. In the intermediate frequency range 


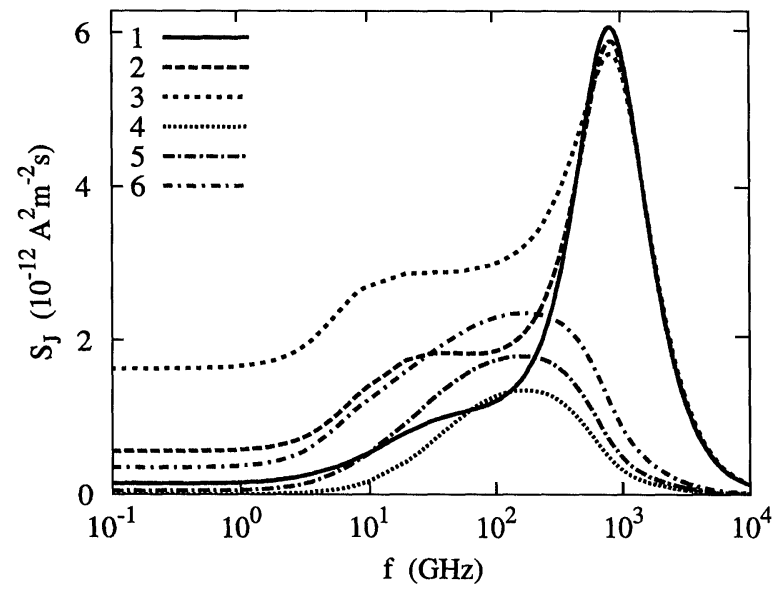

FIGURE 4 Spectral density of hole (curves 1 to 3 ) and electron (curves 4 to 6 ) current fluctuations calculated with the GTF method at, respectively, $U_{d}=0.6,0.65,0.7 \mathrm{~V}$ applied to a bipolar $0.3-0.4 \mu \mathrm{m} \quad \mathrm{Si} p^{+} n$ diode with $p^{+}=10^{17} \mathrm{~cm}^{-3}$ $n=5 \times 10^{15} \mathrm{~cm}^{-3}$.

( $f=10 \div 100 \mathrm{GHz}$ ), a second noise plateau is exhibited by holes while electron current noise show a peaking behavior probably corresponding to a returning carrier mechanism. At high-frequencies we have found a peak of the hole current noise due to plasma oscillations $(f \approx 1 \mathrm{THz})$.

\section{CONCLUSIONS}

We have presented a GTF method based on microscopic noise sources related to velocity, energy, and higher moments change during single scattering events as a unifying scheme able to interpret noise in deep-submicron semiconductor devices. By combining the new noise sources with the transfer fields, the spectral density of the fluctuations of any macroscopic quantity can be represented in a form similar to that given by the standard impedance field method, i.e., as the convolution in real space of the noise source with a transfer field. The numerical results obtained here for various two-terminal devices evidence that the GTF method offers a natural way to include spatial correlations in noise calculations of submicron devices under various biasing conditions.

\section{Acknowledgments}

Authors acknowledge the support of the NATO collaborative linkage grant PST.CLG.976340, the high-level grant DRB4/MDL/no 99-30 of the french Ministere de l'Education nationale, de la recherche et de la technologie, the french lithuanian bilateral cooperation $n$. 5380 of french CNRS, the Galileo project n. 99055, and the project PB971331 from the DGICYT.

\section{References}

[1] Shockley, W., Copeland, J. A. and James, R. P., In: Quantum theory of atoms, molecules and solid state, Ed. Lowdin, P. O., Academic Press (New York, 1966), p. 537.

[2] Van Vliet, K. M., Friedman, A., Zijlstra, R. J. J., Gisolf, A. and Van der Ziel, A. (1975). J. Appl. Phys., 46, 1804; ibidem 1814.

[3] Starikov, E., Shiktorov, P., Gružinskis, V., González, T., Martín, M. J., Pardo, D., Reggiani, L. and Varani, L. (1996). Semicond. Sci. Technol., 11, 865.

[4] Shiktorov, P., Gružinskis, V., Starikov, E., González, T., Mateos, J., Pardo, D., Reggiani, L. and Varani, L. (1997). Appl. Phys. Lett., 71, 3093.

[5] Shiktorov, P., Starikov, E., Gružinskis, V., Reggiani, L., González, T., Mateos, J., Pardo, D. and Varani, L. (1998). Phys. Rev. B, 57, 11866. 

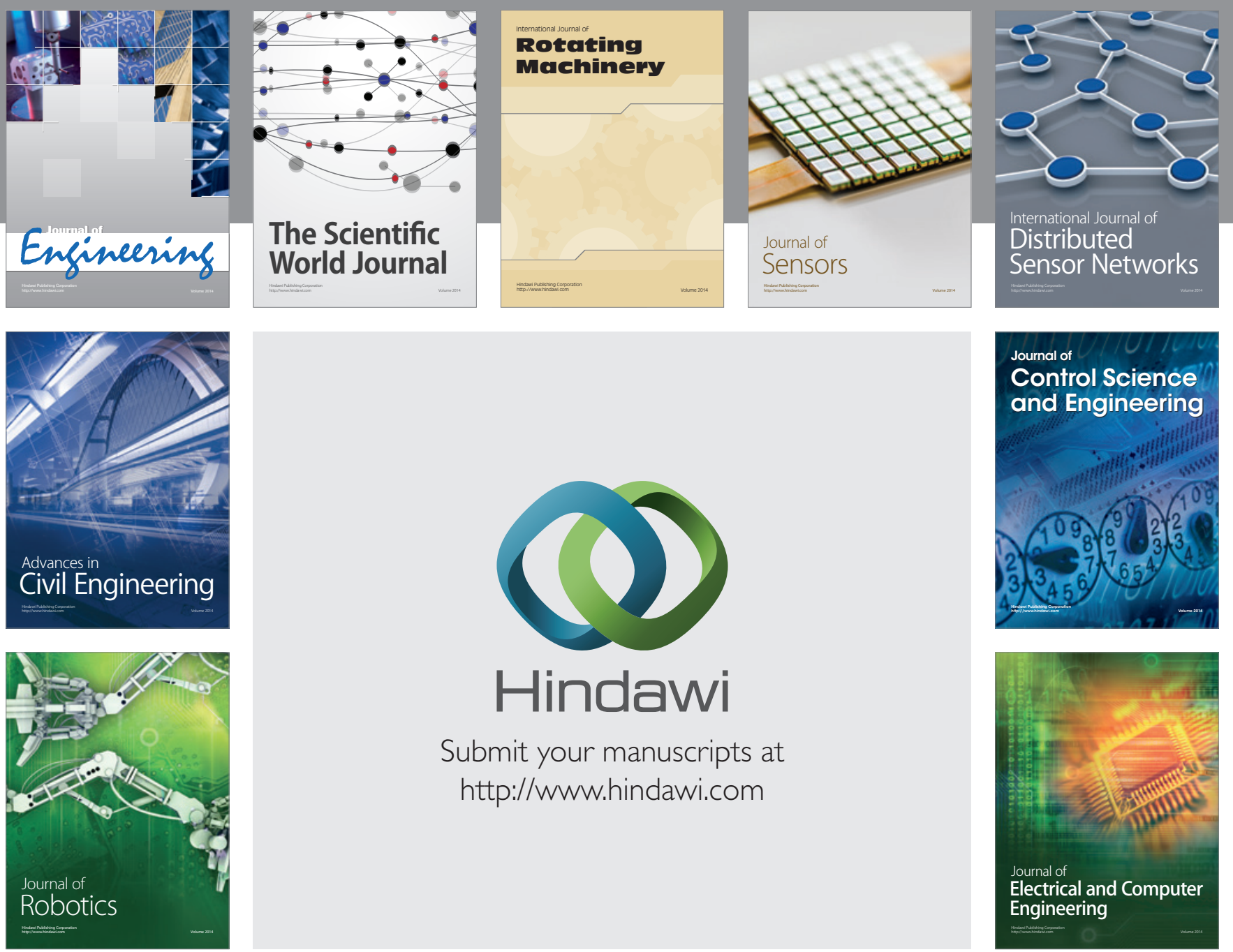

Submit your manuscripts at

http://www.hindawi.com
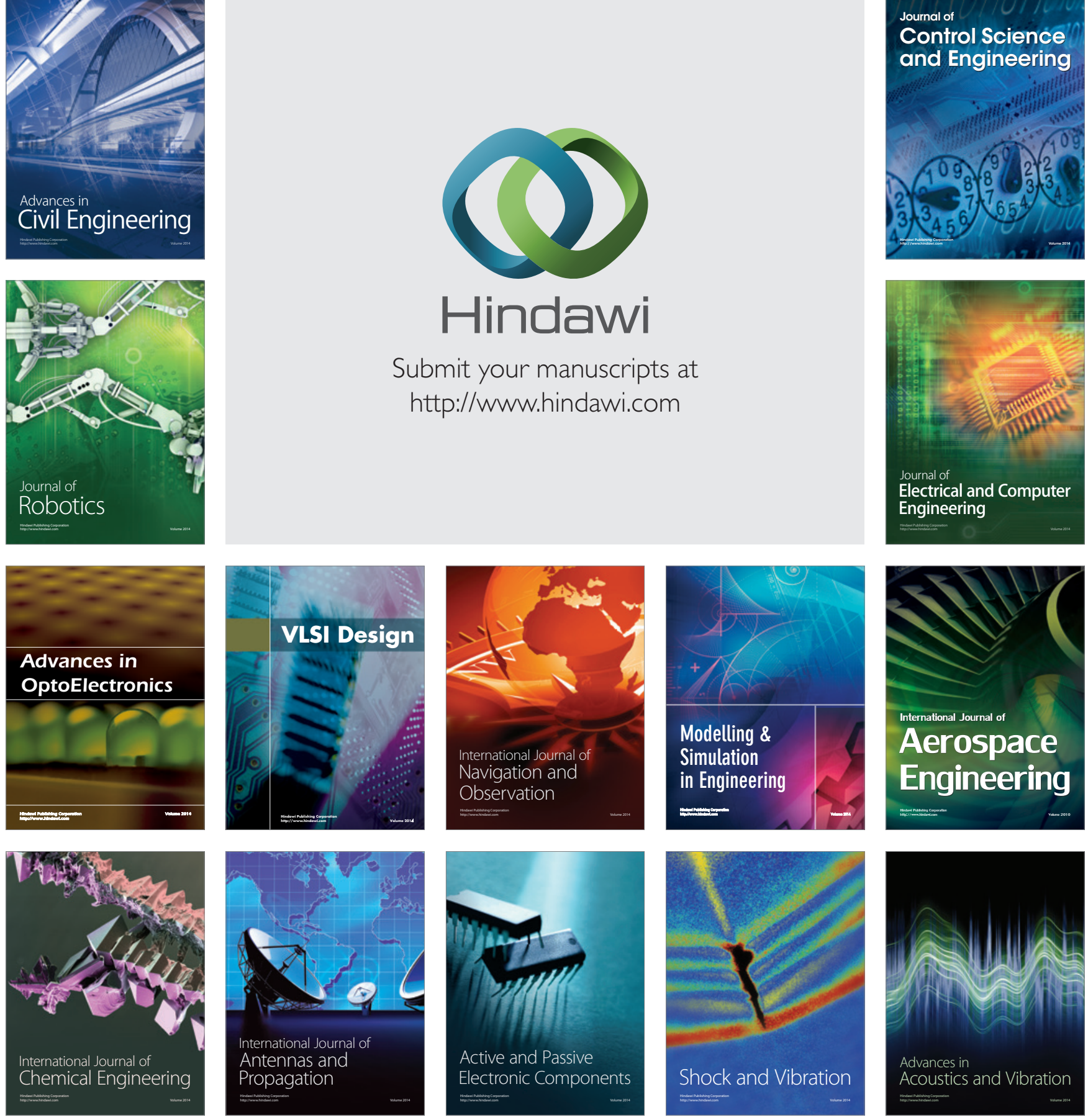